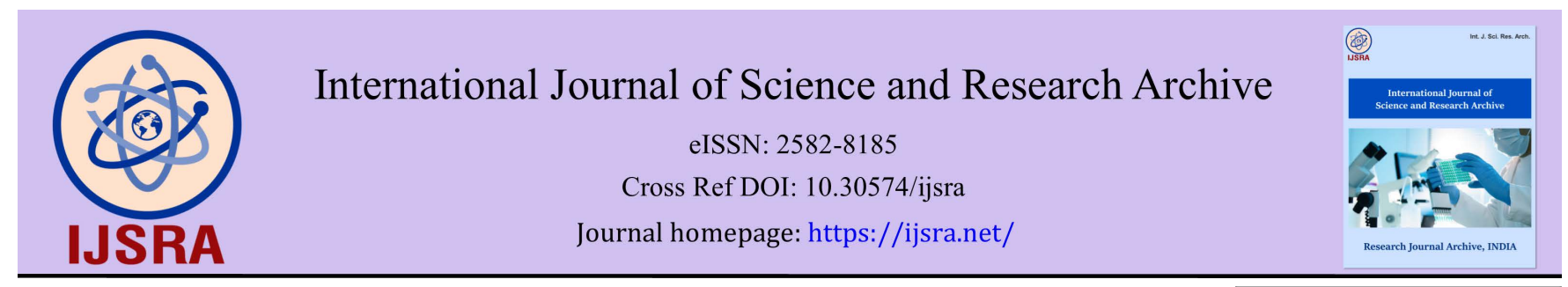

(RESEARCH ARTICLE)

\title{
Co-infection malaria and HIV/AIDS: Epidemiological and clinical aspects at the Communal Medical Center (CMC) Miniere, Guinea
}

Boubacar Siddi Diallo ${ }^{1,}{ }^{*}$, Boubacar Alpha Diallo ${ }^{1}$, Ibrahima Conte ${ }^{2}$, Oumar Diawara ${ }^{1}$, Maechail Jackson Mbobda Ngnetchio ${ }^{1}$, Abdourahmane Diallo ${ }^{2}$, Ibrahima Sory Balde ${ }^{2}$, Telly Sy ${ }^{2}$, Yolande Hyjazi ${ }^{1}$ and Namory Keita ${ }^{1}$

1 University Department of Gynecology Obstetrics, Donka National Hospital, Conakry Guinea

2 University Department of Gynecology Obstetrics, Ignace Deen National Hospital, Conakry Guinea

International Journal of Science and Research Archive, 2021, 02(02), 099-104

Publication history: Received on 23 February 2021; revised on 28 March 2021; accepted on 31 March 2021

Article DOI: https://doi.org/10.30574/ijsra.2021.2.2.0045

\begin{abstract}
Objectives: to calculate the frequency of malaria and HIV co-infections, to describe the socio-demographic characteristics, to describe the clinical signs and to plan treatment.

Methodology: This was a retrospective descriptive study lasting 09 months from August 01, 2019 to April 31, 2020 , conducted at CMC Minière (2nd level reference structure in Guinea). We carried out an exhaustive inventory of cases according to the inclusion and non-inclusion criteria. After applying the selection procedure (inclusion and noninclusion criteria), we obtained a sample of size $\mathrm{N}=68$ cases.

Results: we recorded 68 seropositive malaria patients out of a total of 1232 , i.e. a frequency of $5.52 \%$. The epidemiological profile was that of a person in the age group of 30-34 years $(26.47 \%)$, Unemployed (47.06\%), out of school (54.41\%) and single (75\%). The reasons for consultation are dominated by fever (69.11\%) and physical asthenia (50\%). Patients were from their homes (92.65\%). The type of malaria was severe associated with HIV1 infection (55.88\%). The majority of patients had an average parasite density (10,000-50000) or 54.41\% and $22.06 \%$ had a CD4 count between 200-499 cells / mm3. The antimalarial and antiretroviral treatment consisted respectively of the administration of quinine $65 \%$ and AZT $+3 \mathrm{TC}+\mathrm{EFV}$ or $54.41 \%$.
\end{abstract}

N.B: $A Z T=$ Zidovudine; $3 \mathrm{TC}=$ Lamivudine; EFV = Efavirenz; $\mathrm{d} 4 \mathrm{~T}=$ Stavudine

Conclusion: Malaria contributes to an increase in the viral load in adults with positive retroviral serology, due to the lymphopenia caused during febrile attacks. This finding suggests an emphasis on malaria in people infected with HIV.

Keywords: Co-Infection; Malaria; HIV / AIDS

\section{Introduction}

Malaria and HIV are two major public health problems nowadays, responsible for several million deaths each year, especially in sub-Saharan Africa. Much work has been published on the potential interactions between HIV infection and Malaria. Malaria attacks lead to a transient increase in plasma viral load, but there is no clear evidence that malaria accelerates the progression of HIV infection [1].

\footnotetext{
* Corresponding author: Boubacar Siddi Diallo

University Department of Gynecology Obstetrics, Donka national Hospital, Conakry Guinea.

Copyright $(2021$ Author(s) retain the copyright of this article. This article is published under the terms of the Creative Commons Attribution Liscense 4.0.
} 
Malaria may increase the risk of HIV transmission, including through injections with contaminated equipment or transfusion of contaminated blood product [2]. The impact of HIV infection on malaria infection is modulated by the degree of immunosuppression of patients, but also increases the incidence of malaria attacks, especially as the immunosuppression is deep, with an increase in the severity of malaria attacks and mortality [3].

In urban areas, it has been shown that the forms of malaria are no longer the preserve of infants, but also affect adults. However, HIV infection is rife in these settings in Africa and mainly affects adults. The role of low malaria transmission as well as the resulting lower anti-malaria premunition has been mentioned. However, the hypothesis of some role for HIV has not been ruled out, as studies seem to point to an aggravation of malaria in the event of co-infection with HIV, particularly among adults, even though most of the available literature contrasts with these results. However, work has shown an interaction between malaria and HIV with regard to the immune response and the roles of cytokines has been clearly demonstrated in the two aggravating factors of malaria or of the proliferation of HIV exist in case of co-infection even if their mechanisms remain poorly understood. Few of the studies on the issue have focused specifically on the association of malaria and HIV infection, particularly in adults [4].

Studies have already shown that infection with Plasmodium falciparum stimulates HIV viral replication through increased production by activated lymphocytes of cytokines (IL- 6 and TNF $-\alpha$ ). Also, it is known that serum HIV-1 viral concentrations are higher in patients with malaria and remain elevated for up to 4 weeks after initiation of antimalarial treatment. It is also accepted that the human immunodeficiency virus (HIV) selectively infects CD4 helper lymphocytes and that the latter are potentially responsible for the induction or orchestration of the entire immune response to the various attacks of the cell. organism [5].

The objectives of this study were to: calculate the frequency of malaria and HIV co-infections, describe the sociodemographic characteristics, describe the clinical signs and plan treatment.

\section{Methodology}

This was a descriptive retrospective study lasting 09 months from August 01, 2019 to April 31, 2020, conducted at CMC Minière (2nd level reference Medical Center in Guinea).

All people who consulted and / or hospitalized for malaria with whom the retroviral serology came back positive and whose age is greater than or equal to 15 years were included in the study.

All the people who were consulted and / or hospitalized for malaria in whom the retroviral serology came back negative were not included in the study.

We carried out an exhaustive inventory of cases according to the criteria defined above.

After applying the selection procedure (inclusion and non-inclusion criteria), we obtained ad sample of size $\mathrm{N}=68$ cases.

The variables studied were:

- $\quad$ Epidemiological: frequency, age, sex, profession, level of education, marital status.

- Clinics: Reasons for consultation, method of admission, types of malaria associated with types of HIV.

- Paraclinical: parasite density, TCD4 lymphocyte count.

- $\quad$ Therapeutics: anti-malaria drugs, antiretroviral drugs.

\section{Results}

\subsection{Epidemiological variables}

- Frequency: During the study period, we recorded 68 seropositive malaria patients out of a total of 1232 , ie a frequency of $5.52 \%$.

- $\quad$ Age: The 30-34 age group was the most affected in our series with $26.47 \%$. The average age of the patients was 26 years with extremes of 15 and 42 years. 
- Profession: The unemployed were the most represented with $47.06 \%$ and the least represented were employees with $10.29 \%$.

- Level of education: The out-of-school patients were the most numerous $54.41 \%$ followed by those with a secondary level of education $54.41 \%$.

- Marital status: The study of this parameter reveals that $75 \%$ of patients were single against $7.35 \%$ of married women.

\subsection{Clinical variables}

- Reasons for consultation: Fever and physical asthenia were the most frequent reasons for consultation in our series with respectively $69.11 \%$ and $50 \%$.

- Mode of admission: In our study, $92.65 \%$ of patients came from their homes against $5.88 \%$ of referrals and $1.47 \%$ of evacuees.

- Types of malaria associated with types of HIV: We recorded $55.88 \%$ cases of severe malaria associated with HIV-1 infection, $30.88 \%$ cases of uncomplicated malaria associated with HIV-1 infection; $2.94 \%$ severe malaria associated with HIV-1 infection; $4.41 \%$ uncomplicated malaria associated with HIV-2 infection,; $4.41 \%$ cases of uncomplicated malaria associated with HIV-1 / HIV-2 infection and $1.47 \%$ cases of severe malaria associated with HIV-1 / HIV-2 infection.

\subsection{Paraclinical variables}

- Parasite density: The majority of patients had an average parasite density $(10,000-5,000)$, ie $54.41 \%$.

- TCD4 lymphocyte count: During our study, 27 of our HIV positive malaria patients achieved a TCD4 lymphocyte count; among them: $22.06 \%$ had a CD4 count between 200-499 cells / mm3; on the other hand 38 of our HIV positive malaria patients did not have a lymphocyte count, ie $55.88 \%$.

Table 1 TCD4 lymphocyte count

\begin{tabular}{|l|l|l|}
\hline T-CD4 lymphocytes & Number & \% \\
\hline Absence & 38 & 55,88 \\
\hline$<200 \mathrm{Cell} / \mathrm{mm}^{3}$ & 8 & 11,76 \\
\hline $200-499 \mathrm{Cell} / \mathrm{mm}^{3}$ & 15 & 22,06 \\
\hline$\geq 500 \mathrm{Cell} / \mathrm{mm}^{3}$ & 4 & 5,88 \\
\hline Total & 68 & 100 \\
\hline
\end{tabular}

\subsection{Therapeutic variables}

- Anti-malaria drugs: during our study, $65 \%$ of our co-infected malaria patients received injectable quinine.

- Antiretroviral drugs: the analysis of this parameter shows that $11.76 \%$ of our HIV positive malaria patients did not benefit from antiretroviral treatment, on the other hand $88.24 \%$ did; among them: $54.41 \%$ were subjected to AZT + 3TC + EFV; $19.12 \%$ were subjected to $\mathrm{d} 4 \mathrm{~T}+3 \mathrm{TC}+\mathrm{EFV}$ and only $14.71 \%$ were subjected to $\mathrm{d} 4 \mathrm{~T}+3 \mathrm{TC}+\mathrm{NVP}$.

Table 2 Anti-malaria drugs

\begin{tabular}{|l|l|l|}
\hline Drug & Number of cases & \% \\
\hline injectable quinine 39 & 57,80 & \\
\hline Artemisinin derivatives & 10 & 14,20 \\
\hline Sulfadoxine / Pyrimethamine & 19 & 28 \\
\hline Total & 68 & 100 \\
\hline
\end{tabular}


Table 3 Antiretroviral drugs

\begin{tabular}{|l|l|l|}
\hline Antiretrovirals & Number & $\%$ \\
\hline Without ARV & 8 & 11,76 \\
\hline AZT+3TC+NVP & 37 & 54,41 \\
\hline d4T+3TC+EFV & 13 & 19,12 \\
\hline d4T+3TC+NVP & 10 & 14,71 \\
\hline Total & 68 & 100,00 \\
\hline \multicolumn{2}{|r|}{ N.B : AZT = Zidovudine; 3TC= Lamivudine ; EFV = Efavirenz; d4T= Stavudine }
\end{tabular}

\section{Discussion}

\subsection{Epidemiological variables}

- Frequency: During the study period, we recorded 68 seropositive malaria patients out of a total of 1232 , i.e. a frequency of $5.52 \%$. This result is close to the frequencies reported respectively by THIAM .M and COLL [6] in Dakar 3.9\% and by COURBIERE.B. and COLL [7] in France 2\%. On the other hand, it is clearly lower than that found by BALDE ISMAILA. [8] at the Donka national hospital, Conakry University Teaching hospital, which was $12.9 \%$. This frequency in our series could be explained by the fact that the communal medical center of La Minière is one of the health centers on the outskirts of the city of Conakry where consultation is poor.

- Age: The 30-34 age group was the most affected in our series with $26.47 \%$. The average age of the patients was 26 years with extremes of 15 and 42 years. This result is similar to that found by DE SOUZA J. and COLL [9] in Cotonou who note a maximum peak of Malaria / HIV in the age group of 30-34 years with a frequency of 32.1\% and an age average age 22. MAYI-TSONGA S. and COLL [10] in Libreville found that the age group $\leq 19$ years was the most represented with $41 \%$ against $12 \%$ for the $30-34$ age group. The high frequency in this age group in our series could be explained by the fact that these age groups constitute the most active segment of the population.

- Ocupation: The Unemployed were the most represented with $47.06 \%$ and the least represented were employees with 10.29\%. This result is consistent with those reported respectively by BALDE ISMAILA. [8] at the Donka National Hospital of Conakry University Teaching Hospital, ie 48.3\% housewives and $6.6 \%$ employees and ROSOLONJATOVO JDC and COLL. [11] at the regional hospital of Antsirabe Antananarivo, ie $59.18 \%$ unemployed.

- Level of education: The out-of-school patients were the most numerous $54.41 \%$ followed by those with a secondary level of education $54.41 \%$. This result is similar to those found by NOUTANEWO E. [12] in the study of the UN / AIDS or $62 \%$ of out of school against 38\% of schooling and ABU-RADDAD U. and COLL [13] which reports $57.6 \%$ of out of school and $28 \%$ of primary education. This predominance of out-of-school people could be explained by the fact that they are more numerous in the general population and have less information on health and on the said diseases.

- $\quad$ Marital status: The study of this parameter reveals that 75\% of patients were single against 7.35\% of married women. This result is similar to those reported by BALDE IS MAILA. [8] at the Donka National Hospital of Conakry University Teaching Hospital and ABU-RADDAD U. and COLL. [13] ie 66.8\% and 65.6\% respectively of single women. It could be explained by the fact that in our society there is more libertine situation among single people.

\subsection{Clinical variables}

\subsubsection{Reasons for consultation}

Fever and physical asthenia were the most frequent reasons for consultation in our series with respectively $69.11 \%$ and $50 \%$. Our result is similar to those found by NOUTANEWO E. [12] in the UN / SIDA study and ABU-RADDAD U. and COLL [13], respectively $76.3 \%$ fever and $73.72 \%$ physical asthenia and $60.4 \%$ physical asthenia and 53, 18\% Fever.

\subsubsection{Mode of admission}

In our study, $92.65 \%$ of patients came from their homes against $5.88 \%$ of referrals and $1.47 \%$ of evacuees. This result is contrary to those found by BALDE ISMAILA. [8] at the Donka National Hospital of Conakry University Teaching Hospital and ABU-RADDAD U and COLL. [13] respectively $63.55 \%$ of his patients came from their home, $15.57 \%$ were 
referred, $22.88 \%$ evacuees, and $34.8 \%$ presented spontaneously in the health facility against $56.8 \%$ referrals and $8.4 \%$ evacuees.

\subsubsection{Types of malaria associated with types of HIV}

We recorded 55.88\% cases of severe malaria associated with HIV-1 infection, $30.88 \%$ cases of uncomplicated malaria associated with HIV-1 infection; $2.94 \%$ severe malaria associated with HIV-1 infection; 4.41\% uncomplicated malaria associated with HIV-2 infection,; 4.41\% cases of uncomplicated malaria associated with HIV-1 / HIV-2 infection and $1.47 \%$ cases of severe malaria associated with HIV-1 / HIV-2 infection.

\subsection{Paraclinical variables}

- $\quad$ Parasite density: The majority of patients had an average parasite density $(10,000-5,000)$, ie $54.41 \%$.

- TCD4 lymphocyte count: During our study, 27 of our HIV positive malaria patients achieved a TCD4 lymphocyte count; among them: $22.06 \%$ had a CD4 count between 200-499 cells / mm3; on the other hand 38 of our HIV positive malaria patients did not have a lymphocyte count, ie $55.88 \%$.

\subsection{Therapeutic variables}

- Anti-malaria drugs: During our study, $65 \%$ of our co-infected malaria patients received injectable quinine. This high percentage could be explained by the fact that quinine is the treatment of chosen in cases of severe malaria or malaria attacks accompanied by vomiting and / or nausea.

- Antiretroviral drugs: The analysis of this parameter shows that $11.76 \%$ of our HIV positive malaria patients have not benefited from antiretroviral treatment, on the other hand $88.24 \%$ have benefited. among them: $54.41 \%$ were subjected to AZT + 3TC + EFV; $19.12 \%$ were subjected to d4T + 3TC + EFV and only $14.71 \%$ were subjected to $\mathrm{d} 4 \mathrm{~T}+3 \mathrm{TC}+\mathrm{NVP}$.

N.B: AZT = Zidovudine; $3 \mathrm{TC}=$ Lamivudine; EFV = Efavirenz; $\mathrm{d} 4 \mathrm{~T}=$ Stavudine

\section{Conclusion}

Malaria contributes to an increase in the viral load in adults with positive retroviral serology, due to the lymphopenia caused during febrile attacks. This finding suggests to focus on malaria in HIV-infected subjects and also to think of the co-infection malaria and HIV infection in front of all patients complaining of repeated malaria attacks despite correct management and vice versa.

\section{Compliance with ethical standards}

\section{Statement of informed consent}

Informed consent was obtained from all individual participants included in the study.

\section{References}

[1] Okomé N M; Boguikouma JB; Kombila M: Opportunistic patients with HIV infection at the Jeanne Ebori Foundation Hospital in Libreville (Gabon). Tropical Medicine: 2006; Vol 66.No 2:Pages: 167-171

[2] Atzori C, Bruno A, Chichino G, Cevini C, Bernuzzi AM, Gatti S, et al. HIV-1 and parasitic infections in rural Tanzania. Ann Trop Med Parasitol 1993; 87:585-93.

[3] 0 O Simooya, R M Mwendapole, S Siziya, and A F Fleming. British Medical Journal 1988; 297 doi: https://doi.org/10.1136/bmj.297.6640.30 (Published 02 July 1988) Cite this as: British Medical Journal 1988; 297: 30

[4] AH Diallo, KI Zerbo G. Sawadogo AB, Guigemde TR .Severe malaria and HIV in adult papients in Bobo-Dioulasso, Burkina Faso med. Trop (March) 2004; 64 (4):345-50

[5] Cheryl Cohen 1, Alan Karstaedt, John Frean, Juno Thomas, Nelesh Govender, Elizabeth Prentice, Leigh Dini, Jacky Galpin, Heather Crewe-Brown. Increased prevalence of severe malaria in HIV-infected adults in South Africa.DOI: 10.1086 / 498023 
[6] THIAM, Martin P 0. Human Malaria Immunology and Cardiology.

[7] COURBIERE.B: HIV infection as a co-factor in adults.Ann Soc 2004; 159-178

[8] Baldé Ismaila. Malaria and HIV infection: epidemiological, clinical and biological aspects in the service of infectious and tropical diseases at the Donka National Hospital. Thesis med. Conakry. Guinea. 2003.

[9] Bertrand Gachot, De Souza, Charlotte Behr. Severe Malaria: The Practitioner's Review 2001; 51:638-43

[10] Charmot G, Simon F, Mayi TSONGA S. HIV infection and malaria. Practitioner Review, 1999; Vol. $40 \mathrm{n}^{\circ} 23$

[11] Bloland PB1, Wirima JJ, Steketee RW, Chilima B, Hightower A, Breman JG. Maternal HIV infection and infant mortality in Malawi: evidence for increased mortality due to placental malaria infection. Author information: AIDS (London, England), 01 Jul 1995; 9 (7):721-726 DOI: 10.1097 / 00002030-199507000-00009 PMID: 7546417

[12] WHO: Department of HIV / AIDS. Situation report. JUNE 2008.

[13] Laith J Abu-Raddad, Padmaja Patnaik, James G Kublin. Dual Infection with HIV and Malaria Fuels the Spread of Both Diseases in Sub-Saharan Africa. Science February 2007; 314 (5805):1603-6. DOI: 10.1126 / science.113233 